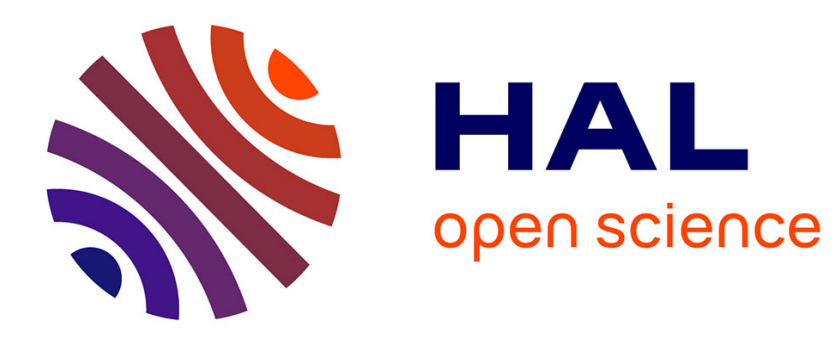

\title{
Un voyage dans l'au-delà, vers l'an mil. Brèves remarques autour d'une apocalypse byzantine
}

\author{
Jean-Marc Rosenstiehl
}

\section{To cite this version:}

Jean-Marc Rosenstiehl. Un voyage dans l'au-delà, vers l'an mil. Brèves remarques autour d'une apocalypse byzantine. Revue d'histoire et de philosophie religieuses, 2000, 80, pp. 93-104. hal00121354

\section{HAL Id: hal-00121354 \\ https://hal.science/hal-00121354}

Submitted on 20 Dec 2006

HAL is a multi-disciplinary open access archive for the deposit and dissemination of scientific research documents, whether they are published or not. The documents may come from teaching and research institutions in France or abroad, or from public or private research centers.
L'archive ouverte pluridisciplinaire HAL, est destinée au dépôt et à la diffusion de documents scientifiques de niveau recherche, publiés ou non, émanant des établissements d'enseignement et de recherche français ou étrangers, des laboratoires publics ou privés. 
Article paru dans la Revue d'Histoire et de Philosophie Religieuses, 2000, Tome $80 n^{\circ} 1$,

Publié avec le concours du CNRS et du Centre National du Livre 


\title{
UN VOYAGE DANS L'AU-DELÀ, VERS L'AN MIL. Brèves remarques autour d'une apocalypse byzantine
}

\author{
Jean-Marc Rosenstiehl \\ ('entre d'Histoire el civilisation de By/ance (UMR 7572) \\ Palais de l'Université -- 67084 Strashourg C'edex
}

\begin{abstract}
Résumé : L'article présénte une' apocalypse byacantine méconnue. La conclusion sommaire somlève la question de la circulation de motifs connus par les écrits intertestamentaire's. L'hypothèse' d'un cheminement par étapes dans le' temps et l'espace (oì la Sirie jouerait un rôle des plus importants) fournirait une explication séduisante à la présence, à première v'ue paradoxale', de motifs orientaux antiques dans des compositions tardives.
\end{abstract}

Abstract: This contribution consists in a presentation of a nearly unknown byzantine apocalypse. A brief conclusion deals with the question of the transmission of themes originating from pseudepigraphic literature. Such themes may have travelled in time and space (Syria plaving there a main role) which would allow to explain the presence of old eastern themes in late literary texts.

À l'exemple de nombreux témoignages écrits de l'Antiquité, c'est peu après l'invention de l'imprimerie que les temps modernes ont vu la première diffusion à grande échelle d'un texte apocalyptique byzantin : les Révélations du Pséudo-Méthode'.

Cet écrit est un bon exemple pour illustrer l'histoire de la recherche dans ce domaine : il faudra attendre la deuxième moitié du 19" siècle pour que paraissent les éditions' et travaux modernes '. Cependant, la plus grande partie des problèmes posés par cette littérature ne trouva guère alors d'explication satisfaisante.

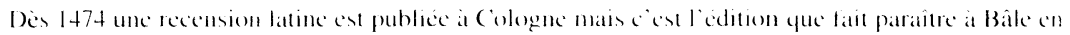

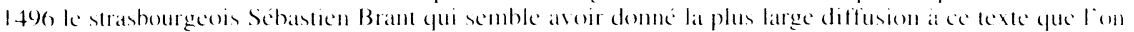
considere comme le decument le plus important de l'apocalyptique by $/$ antinc.

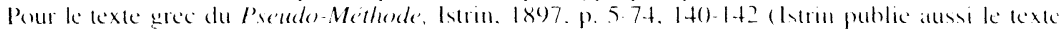

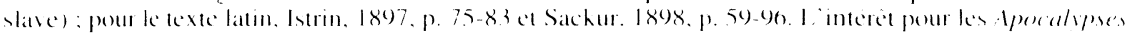

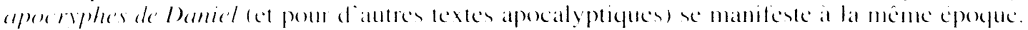

Les commentates se multiplient dams les dernieres decemies du siecle et notamment con Allemagne

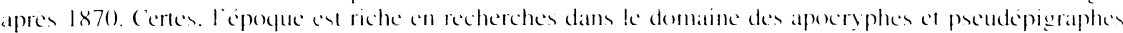

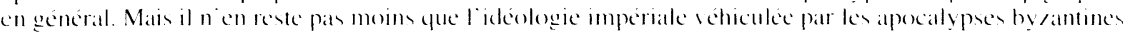

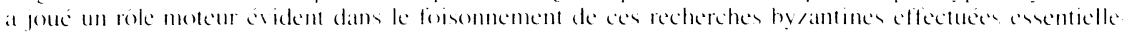

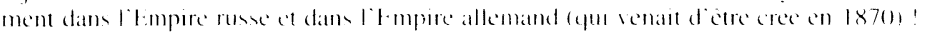


Bien plus tard, ke travail d’un savant hongrois ferat date' : en étudiant un texte syriaque qu`il avait découvert. il apporte d’abord la démonstration d'une hypothese ancienne" selon laquelle le PséladoMéthode n’avait pas été composé èn gerec, mais yu il était la traduction dans cette langue d'un original syriaque. Il avance ensuite une hypothèse de travail : l'écho de traditions iraniennes que l'on peut déceler dans les Rélélations s'explique par l'origine de l'auteur ; celui-ci ne vivait pas en Syrie, mais en Mésopotamic, qui, à l'époque sassanide, était une province de l'empire perse.

Après la mort de M. Kmosko, il faudra attendre plus de cinquante années pour voir parâtre enfïn l'original syriaque . Et ce n’est qu'au cours de la dernière décennic qu'on a vu ce texte, ainsi que ses versions principales, édité à frais nouveaux". Qui plus est, des éditions récentes permettent aussi l'acè̀s facile à des écrits appartenant au même genre littéraire de ce que l'on pourrait appeler par commodité les « apocalypses historiques". »

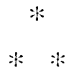

Un autre genre d'apocalypses commence à intéresser les critiques : elles ont pour objet la relation d'un voyage dans l'Au-delà. Une bonne part de ces textes produits par l'Occident médiéval ont été commodément réunis dans une excellente petite anthologie "1. Pour l'Orient, malheureusement, on ne dispose pas d'un tel outil et les matériaux restent dispersés, isolés et relativement peu diffusés et connus.

Je voudrais m'attarder ici sur un de ces textes, une Rélélation du Ciel ét des Châtiments publiéc il y a un centaine d'années, d'abord par L. Radermacher", puis par R. Homburg "La présentation qui suit en résume, à grands traits, le contenu :

Kmosho. 1931. If ne s'agit pas d'un articke, mais d unc conference knue a Vieme ldans ke cadre de la 6 Journéc allemande des ()rientalistes) ke 13 juin 1930 ou Kmosko brosse en quelque sorte ke programme-cadte de ses recherches. Il reservatit ses demonstrations pour une monographic qui no est jamais parue (Kmosko est mort en 1031).

Le Vaticanus Syrus 58.

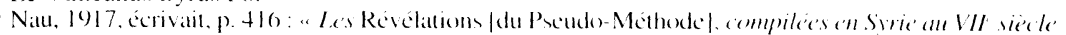

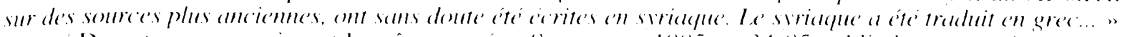

Deux travaux paraissen la meme annee : Stermamn, 1985, p. 34-8.5 publie ke texte syriaque avec une traduction allemande: Akexander, 1985. dome une raduction anglaise, p. 36-51. precédée d'une introduction. p. 13-33.

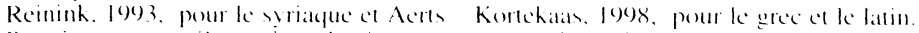

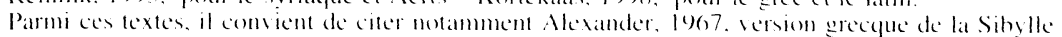
de Tibur: Berger (1976), apocalypse grecyue de Daniel: Verhelst, 1983. preude-1 phem: Ryden (1974)

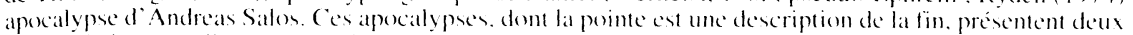
caracteristiques: deune part. elles compontent des compilations de themes apocalyptiques orientaux

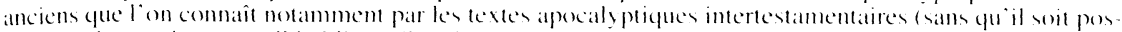

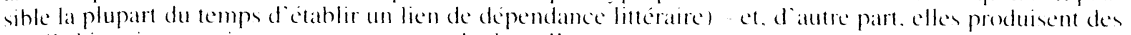
details historiques qui permettent souvent de dater liexure.

Dinzelbacher, 1989. Voir atussi la riche ctude de Caro/si. 1994. sur ce theme dam ke monde latin.

Radermacher, 1898 : a partir d un manuscrit unique ed acephale de Paris: Pintroduction et les

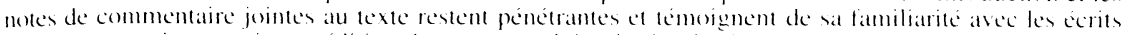

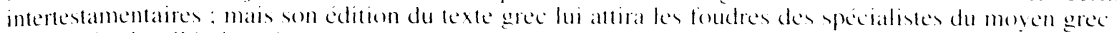

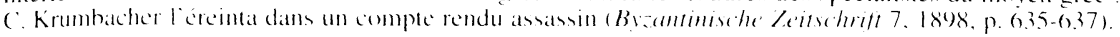




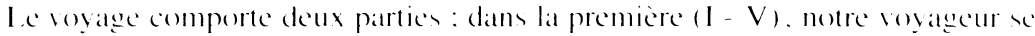
cantonne essentiellement dans le roke deun obsersateur prespue statigue en décrit der situations ed des événements célesters.

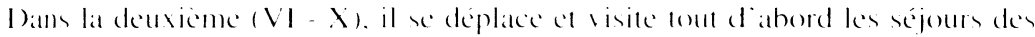

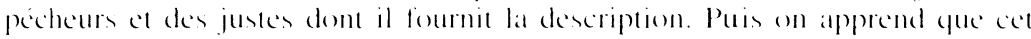
homme arrivé du monde terrestré a y étre remogé dans le but de lémoigner de cir yu il a vu dams l'Au-dela.

Dans une breve conclusion (XI XII). enfin, il reçoit des instructions pour ke retour yui font lohjed de la lin du récit.

An commencemem du recit.

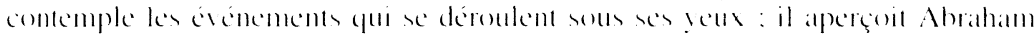

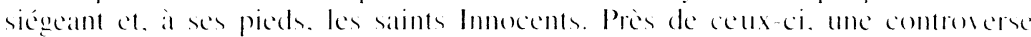
anime un groupe de femmes dont trois demandent à Died de châtier ker

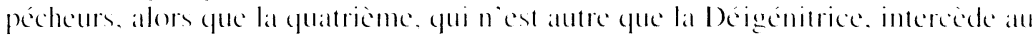
contrate pour les hommes "e demande a Dieu de ne pas prosequer leur perte (1)

lat sépuence suisante permet atu voyagere de voir ke Trone divin aved son entourage angéligue célébrant la liturgie céceste (II - III).

Ensuite il découve le paysage environnant, à travers les sept portes descieux, dont chacune possède une caratéristigue diférente (IV).

Retour a la controverse du début entre les saintes femmes, devant ke Trone, ou lon détaille les griefs : sainte Kyriake dénonce les pécheurs qui par leurs entrates at lat chastete ou leurs aceupations manuelles me respectent pas ke jour

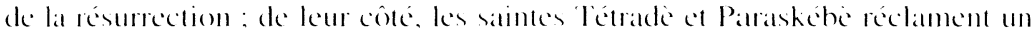
chattiment exemplaire pour ceux qui les souillent par ke non-respect du jeune du merceredi et du vendredi. notamment par l'absorption de viande el de fio-

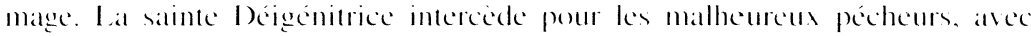

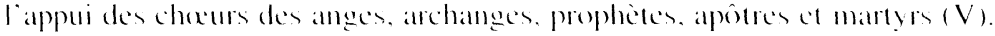

Apres cetle premiere partic consistant essenticllement en un spectate e. commence alors, sous la conduite d'un ange accompagnateur, une visite des lieux des justes ét des pécheurs.

laxcursion amene vers les endrois habiluels, fleuve de leu, ver qui ne dorl pas. où sont précipités des pécheurs liäes et ordinaires, puis près de fours ronflants ou de puits profonds ou de's ecclésiastiques, pretres el diacres, grillent ou croupissent ( VI).

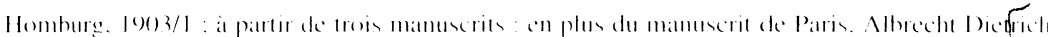

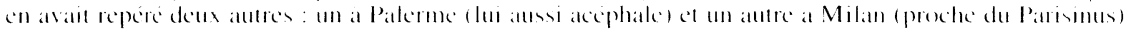

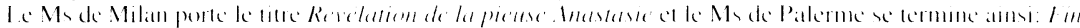

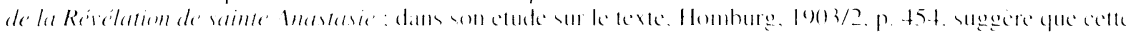

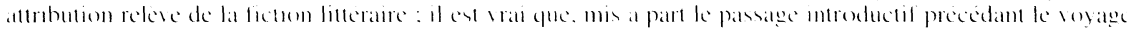

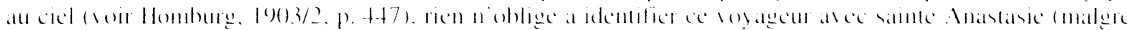

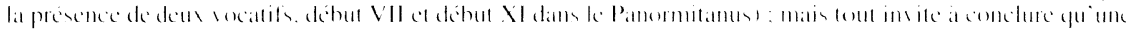

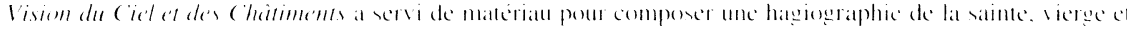

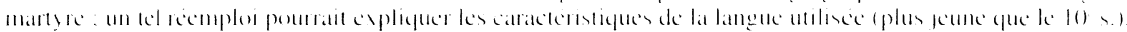

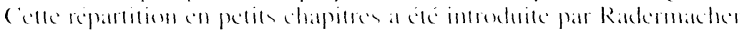

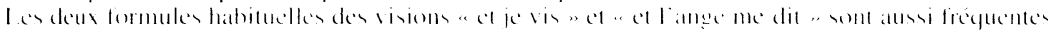

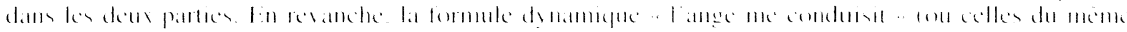
1y

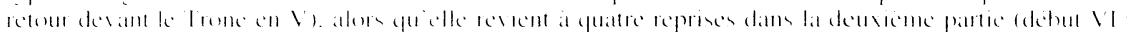
fin VI debul VII : dehul VIII : tin I. I

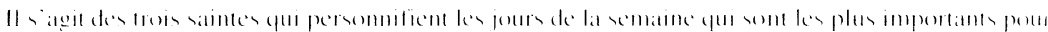

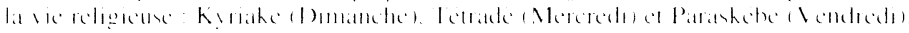

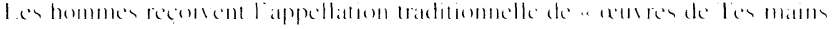

l.

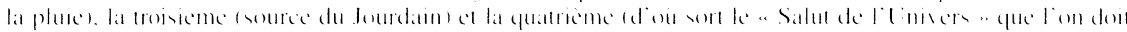

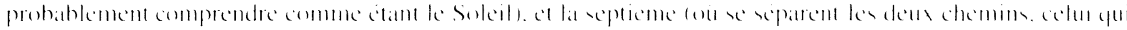

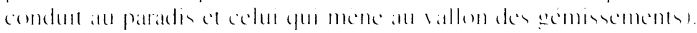

Vor ci-k 


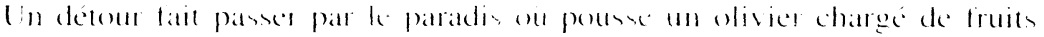

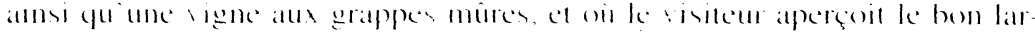

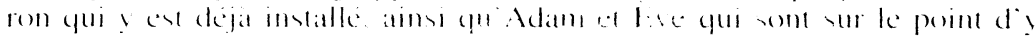
entrel ( VII)

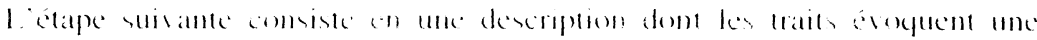

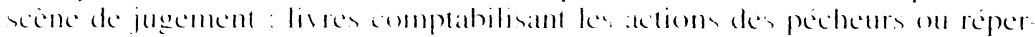

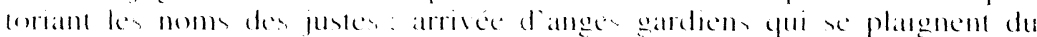

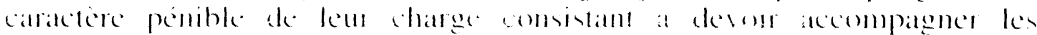

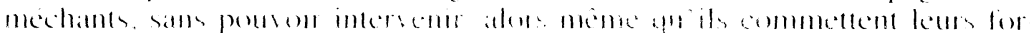
filits (VIII).

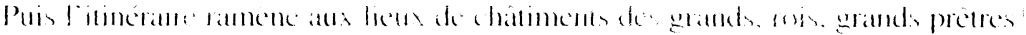

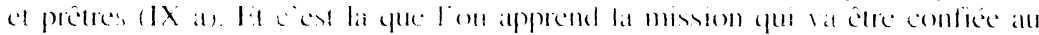

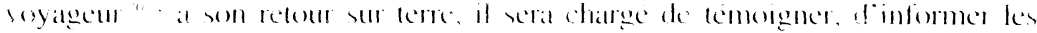

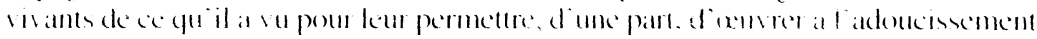

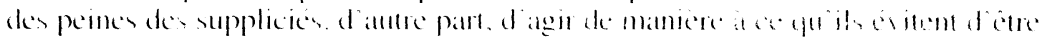

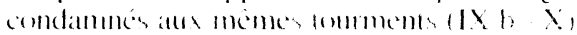

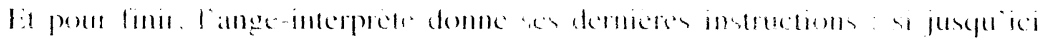

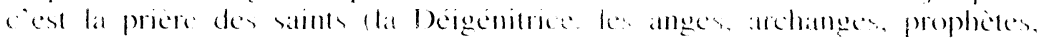

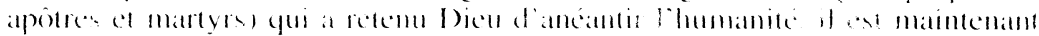

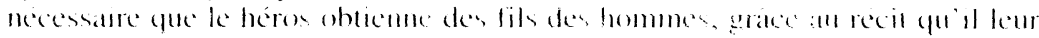

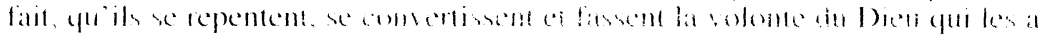
crés at son image (XI).

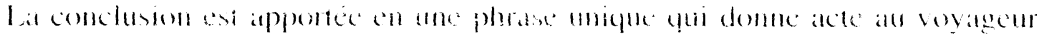

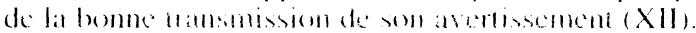

Celle breve analyse suffil a monter que pour ce qui concerne sa forme, ce voyage dams I'Audela porte les signes d"unte facture lout a fail classique

Pour ce qui est de la date de composition an pluter, de compilation - les motilis proprement religieux ne domnent guère que des indications générales: ainsi la présence (en 1 et $V$ ) de sainte Kuriakè. personnification du Dimanche. des saintes Tétrade a Paraskébè, per. sonnifications du mercredi el du vendredi". joure les plus importants de la semaine, parce qu'ils sont jeûnés saat én eertaines périodes de l'année liturgique ici Pentecôte et Dodékahèméron) ne permet guère de préciser de date " De même lat formule: «Tenons-nous bien!

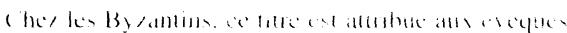

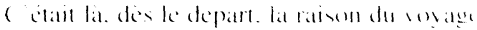

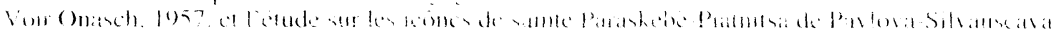
$11406)$

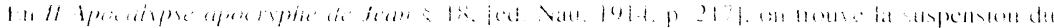

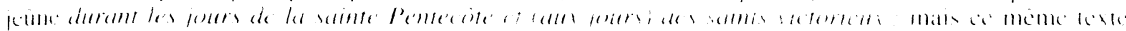

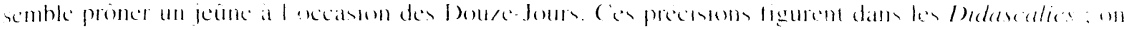

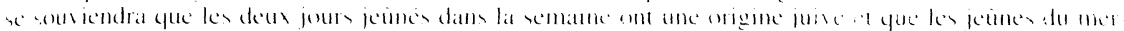

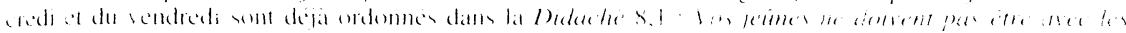

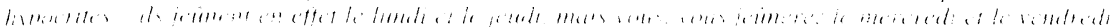


Tenons-nous dans la crainte ! » qu'une voix (céleste) clame à l'ouverture de la liturgie angélique autour du Trône (en III) est celle-là même qui ouvre le dialogue eucharistique dans les liturgies byzantines:".

A l'exemple de nombreux textes apocalyptiques byzantins, ce ne sont pas les motifs qui permettent de dater l'ouvre. En revanche, il n'est pas rare, dans cette littérature, que l'auteur introduise des indications historiques assez précises". Et c'est aussi le cas ici : lors de la visite des lieux de châtiment des grands (rois, évêques, en IX), le voyageur assiste à une scène clairement datée ; on y voit l'empereur Nicéphore Phocas reprocher à son général Jean Tzimiskès de l'avoir assassiné alors qu'ils avaient conclu entre eux une alliance de paix en "posant les mains sur sainte Sophie »; Tzimiskès, le parjure usurpateur n’a pas profité de sa félonie et n’a pas été jugé digne de compassion et ne siège donc pas, dans l'Au-delà, sur un trône impérial, selon ce que nous rapporte notre voyageur. Or, on sait que Jean Tzimiskès, après avoir fait assassiner Nicéphore Phocas en décembre 969, exerça le pouvoir à peine six ans et mourut du typhus en janvier 976; c'est donc au seuil de l'an mil que doit se situer cette dispute dans l'Au-delà et c'est aussi fort probablement la date de composition du texte ${ }^{25}$.

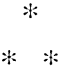

Vers la fin de l'apocalypse, le voyageur est renvoyé sur terre, chargé d'une mission assez précise : il doit annoncer ce qu'il a vu dans l'Audelà à la famille et aux proches de deux pécheurs rencontrés dans les tourments, et leur demander de distribuer aux pauvres les biens (injustement acquis) pour permettre à ces pécheurs de bénéficier d'un sort adouci, d'une part, et, d'autre part, pour que leur famille, après la mort, ne coure pas le risque de finir dans les mêmes châtiments (IXb et X) ${ }^{26}$.

Ce passage évoque d'emblée un petit conte oriental que l'évangéliste Luc met dans la bouche de Jésus. On connaît ce texte -- unique et assez singulier dans tout le Nouveau Testament - sous le titre de Parabole du riche et du pauvre Lazare". Dans les châtiments infernaux.

Cependant, rien n'exclut yu une telle formule, adressée à l'assistance au momemt ou te prêtre va presenter l'offrande. né soil qu' une survivance d'une ancienne liturgie juive

Voir ci-dessus la fin de la note 9

Sur cette date, voir déjà (idel. 1871. 1) autres éléments peuvent étre invoques pour confirmer cette date : ainsi, un autre personnage dans les tourments décline son identité : orginaire de (Corinthe, ce fonce tionnaire de haut rang se nomme Pierre et occupe la charge de protospathare : Radermacher. 1898, p. 11 12, veut lidentifier à un contemporain de Thimiskes et Homburg, 19(1)3/2, p. 445-4.47. songe à Pierre

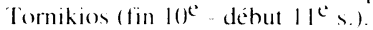

La mission est ensuite élargice el vise l'ensemble de l'humanité : le voyageur doit amener les fils des hommes a la repentance el a lat conversion pour que decu ne les ancantisse pas et ne les fasse pas disparaitre de la surface de la terre (XI). (éte généralisation est fort probablement secondaire.

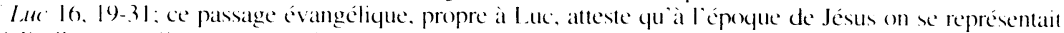
l'Au-dela d'une maniere assé précise : plus particulièrement ici. en $I$ du lo. des châtiments et des récom penses, qui constituent une partic classique des apecalypses du type " voyage au ciel ". Marc 13 et ees paral

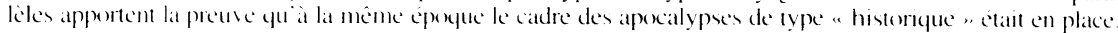


le riche demande à Abraham, dans un premier temps, de lui procurer un adoucissement de situation, et, dans un deuxieme temps, de bien vouloir renvoyer Lazare dans le monde pour que les membres de sa famille, instruits par le témoignage d'un revenant, se convertissent et ne viennent pas, eux aussi, dans ce lieu de toument.

À premiere vue, il est très tentant d'avancer une solution facile: l'apocalypse byzantine a pour source directe la parabole évangélique. Mais un coup d'acil plus précis invite à une grande prudence. En effet. si la situation mise en scène dans les deux textes latil apparaitre une certaine similitude, la plupart des signes extérieurs attestant un emprunt ou une dépendance font défaut : sur ke plan de la forme, les personnages ne sont pas les mêmes, la trame est diférente, le style est autre. le vocabulaire n'est pas identique. be surtout, pour ce qui est du fond, la chute du conte de l'Évangile est à l'exalet opposé de ecelle de la vision byzantine : dans Late $(16,31)$, la chute est brutale : "s"ils n’écoutent pas Mö̈se et les prophètes, même si quelqu'un d'entre les morts ressuscite, ils ne se latisseront pats convaincre! " Tout autre est la lin de l'apocalypse (IXb el X) : dés que l’ange présente le voyageur aux pécheurs dans les châtiments, il annonce la suite : " (elui-ci (vient) du monde de vanité et je men vais l'y faire retourner!"

Certes, s’il n’est pas possible de conclure à une dépendance littéraire, il n’en reste pas moins une parenté entre les deux textes alu plan des motifs. Et eette rencontre n'est pas fortuite; des recits de voyages dans l'Au-dela existatent il y a deux mille ans ; et on imagine sans peine Jésus utilisant les motifs habituels de tels récits, mais en leur donnant un sens inhabituel et nouveau, en en détoumant, voire en en renversant la chute.

Plusieurs motifis apparaissant dauns l'apocalypse byzantine présentent un interêt particulier; je voudrais mattarder quelque peu sur deux d'entre eux".

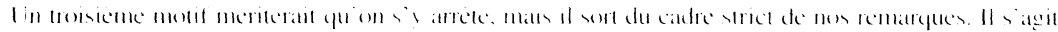

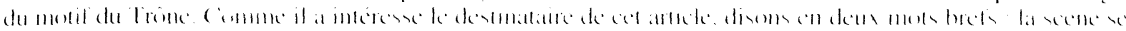

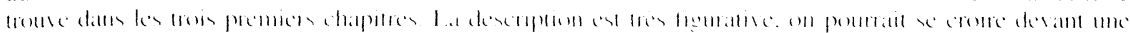

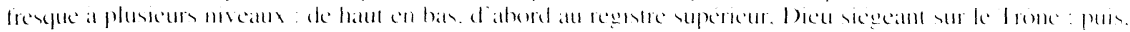

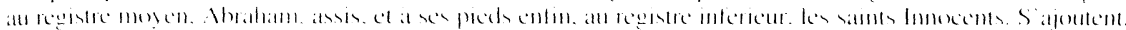

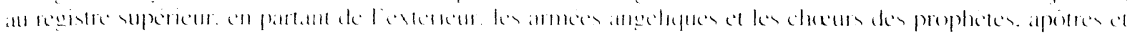

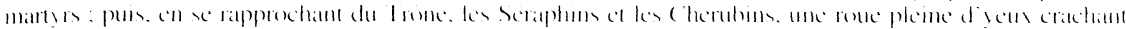

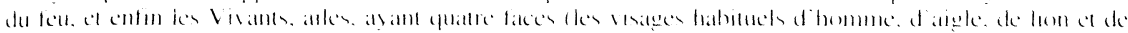

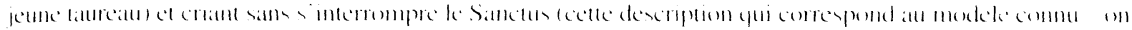

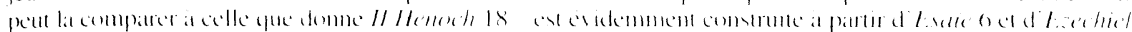

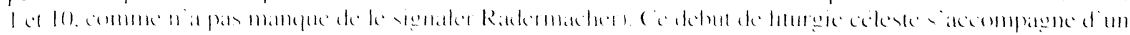

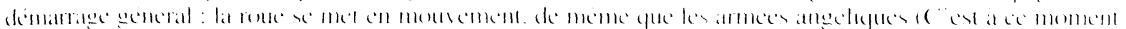
la qu

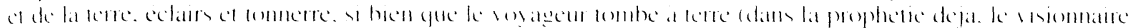

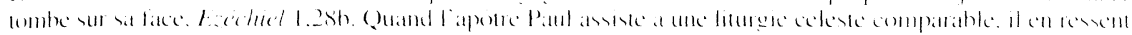

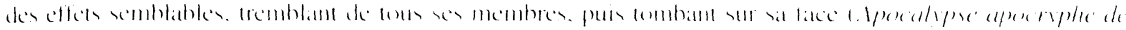

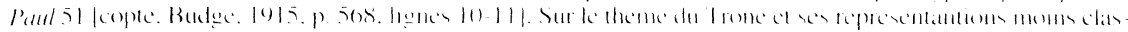

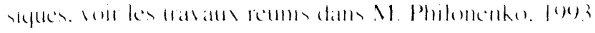


I. Un premier motif ne manque pas d'attirer l'attention. Le voyageur voit devant lui. l"une après l"autre, sept portes du ciel". Au passage de la quatrième porte, qui est probablement celle du Soleil, il aperçoit des oiseaux gigantesques. L’ange explique, d'une manière tout à fait intelligible, la fonction de ces oiseaux, qui est double: d'une part, ils accompagnent le Soleil et, au moyen de leurs ailes, filtrent ses rayons brûlants pour que la terre ne soit pas incendiée ; et d'autre part. ils se mettent aussi à voler lorsque tombe la pluie, pour que la terre ne soit pas noyée sous les eaux.

Ce motif n’est pas inconnu dans les textes byzantins : on le trouve dans le Débat de l'Azymite ét du Panagiote" "Mais on le rencontre déjà dans les pseudépigraphes juils. Par exemple, èn $I I I$ Barach 6, où il s'agit d'un seul oiseau, identifié au Phénix, qui joue le rôle de " prote'teur de la terre habitée ${ }^{31}$ " en filtrant, au moyen de ses ailes déployées, les rayons du soleil, permettant ainsi la survie de la race des hommes et de toute espèce vivante. Ensuite en $/ / H e ́ n o(h 12$ qui parle de deux esprits volants, de la forme de deux oiseaux (le Phénix et le Chalkédri) "qui portent" la rosée et la chaleur.. ". À première vue, le rapport entre la rosée et la chaleur n'est pas clair dans ce texte. On a pu essayer de comprendre ce passage en suggérant une complémentarité entre la rosée et la chaleur : la rosée servirait à protéger la terre de la chaleur ". L'apocalypse byzantine y voit en revanche une succession sans aucun lien de complémentarité : les rayons du soleil doivent être filtrés pour ne pas brûler les êtres vivants sur terre; et de même la chute dangereuse de la pluie doit être filtrée pour ne pas noyer les habitants de la terre ${ }^{3-4}$. Et nous pourrions bien avoir là la véritable explication du passage.

On avait identifié le motif de ces oiseaux célestes dans la littérature indienne ${ }^{35}$, et pour le passage de $I I I$ Baruch, rapproché le mythe antique du Phénix ". Les gigantesques oiseaux célestes de l'apocalypse byzantine sont plusieurs - leur nombre n’est pas précisé. Et je ne serais pas surpris que leur nombre ait pu atteindre trente, auquel cas

Le texte est en matuvatis état, voir ci-dessus, note 17.

A. Vassilies, 1893, p. I85 (rapprochement rekeve déja par Radermateher, tout comme les deux suivants).

ho phulax tés oikonméness, habituellement traduit par "gardien ". ce qui n'est pas très heureux: "protecteur" conviendrait bien mieux.

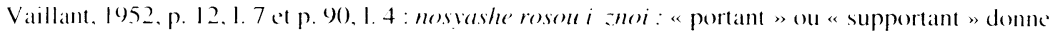
un meilleur sens ici que la traduction de Vatllant "apportant lat rosée et la chaleur ". qu'il convient probablement de corriger dans Vaillant. 1952. p. 13 et 91 et Dupont-Sommer - Philonenko, 1987, p. 1179.

("est lexplication avancée, avec une certaine prudence ( sans doute "), par M. Philonenko dans Dupont-Sommer - Philonenko, 1987. p. 1179, note sur XII.I. F.I. Andersen, dans ('harkesworth. I983. wol. 1. p. 122 note 12a, a senti la difficulti expligue : toutes les versions latissent dans liobscurité cer-

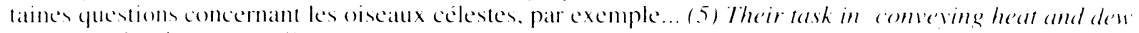

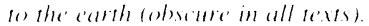

II n' y a guere qu en tggypte ou l eau qui tombe du ciel soit considérée comme maléfique (la bonne eau vient des cours d'eatu. du Nil, bien entendu) : un tel détail dénonce son orignine égyptienne.

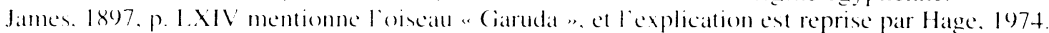

le málange des deux motifs ess une particularité de III Barme hou lioiscau céleste est unique, alors yue dams les deux kexters il y ena deux or plus. 
il faudrait les rapprocher d'un motif iranien fameux, celui de l'oiseau céleste gigantesque

II. Un deuxième motif vaut qu on s'y arrête. Après la description du tableau céleste autour du Trône de Dieu, on voit l'ange enlever le visionnaire pour lui montrer les châtiments. La premièrétape (en VI) conduit auprès du "ver qui ne dort pas " lequel, précise le texte, "était le fleuve au bord duquel se tenait une multitude d'hommes et de femmes ". Ce fleuve de feu doit être traversé ; un groupe de pécheurs savance et alors " les vents fusèrent et le pont se retourna comme aspiré par un vent violent : et les pécheurs tombaient comme les leuilles des arbres. "

La mention du Pont n'a rien de surprenant dans une apocalypse médiévale. En Occident, les exemples vont de Grégoire le Grand "jusqu'au chevalier Tondale ". Dans la littérature intertestamentaire le motif est bien attesté " ; et on a trouvé dans les fragments de Qoumrân une attestation de ee "pont de l'Abîme", qui est un des thèmes centraux de l'apocalyptique iranienne ". Si le motif est bien connu, le traitement que ce pont inflige aux pécheurs est original : les vents secouent le pont comme une branche et les méchants en tombent comme des feuilles mortes.

Ces exemples nous amènent à formuler quelques esquisses de conclusions. Que des motifs orientaux anciens aient été utilisés par les compilateurs d'apocalypses byzantines coule de source. L'emploi de motifs iraniens ne fait aucun doute. tant dans les apocalypses du type historique que dans celles de la typologie du voyage dans l'Au-delà. Doit-on dès lors conclure à une influence directe, iranienne, en supposant par exemple. un lieu de composition situé dans la sphère d'influence de lempire perse musulmane cette fois, en proposant de localiser le compilateur dans une rone d'influence musulmane" "?

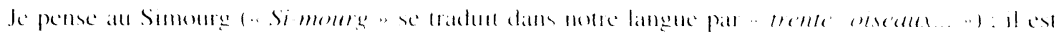

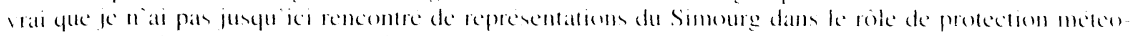

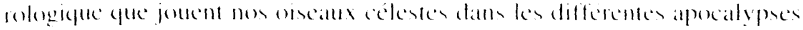

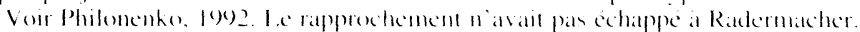

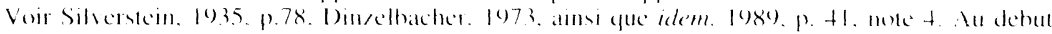

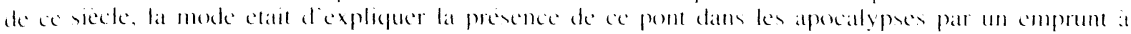

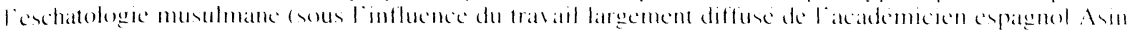

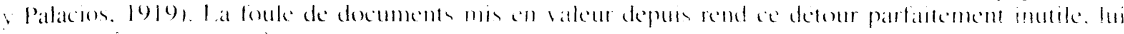

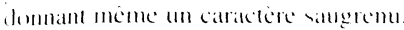

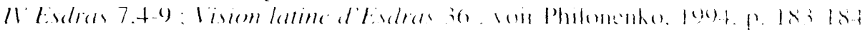

$11(152 \%$, wow Philomenho, 194. P 182.

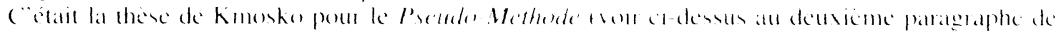

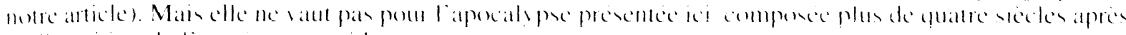
la dispartion de lemple semathide.

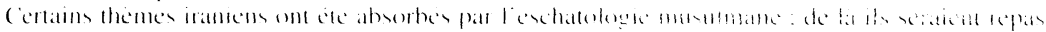

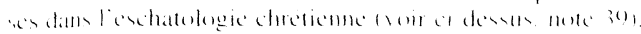


Lexemple du pont Cinvat montre que cela n'est pas une question d'influence directe, ni même de lieu de composition dans la sphère d'influence. L'attestation de ce pont dans les représentations qoumrâniennes change les données du problème : des motifs iraniens sont présents dès l'origine de l'apocalyptique ${ }^{+1}$ et le dossier des influences iraniennes sur la secte de Qoumrân prend de plus en plus de consistance et d'importance ${ }^{15}$. Par la suite, ces motifs semblent disparaître pendant un certain temps, pour reparaître on ne sait trop ni pourquoi ni comment ${ }^{\text {th }}$. Il est ainsi de fait impossible de reconstituer une chaîne littéraire continue.

Il n'y a donc pas lieu de chercher une solution qui règlerait comme par magie tous les problèmes. Mais en considérant certaines étapes parcourues par un motif au cours de dix siècles, on ne peut résister à la tentation de poser au moins une hypothèse de travail. Et en l'occurrence, une telle hypothèse reposerait sur l'idée d'un cheminement géographique dont voici les grandes lignes :

Les motifs apocalyptiques juifs, puis chrétiens, voire même les textes entiers, après s'être développés en Palestine (en hébreu ou en araméen), en Égypte ou à Alexandrie (en langue grecque pour la plupart), se sont répandus dans un espace géographique de plus en plus étendu, notamment en Syrie ${ }^{17}$, où ils ont, semble-t-il, été traduits en langue syriaque et où ils ont continué leur vie ${ }^{4 x}$, alors même que les originaux ont pu disparaître sans laisser de traces.

À son tour, au cours des siècles suivants, ce conservatoire apocalyptique syrien aurait servi de réservoir auquel ont puisé les auteurs d'apocalypses portant une marque plus tardive, d'époque byzantine. L'apocalypse grecque, au centre de cette étude, a fort bien pu pêcher ses motifs dans ce réservoir syriaque ${ }^{+1}$.

A coté du Pont Cinvat dans un fragment qoumrânien, on rencontre d'autres motifis eschatologiques iraniens dans les écrits intertestamentaires ; le plus frappant est, sans conteste. celui de la daena identifié par Böklen. 19(1)2, et Widengren, 1957, p. 238, n. 3, dans le Téstame'nt d'Abraham A 17,6-8, voir Schmidt, 1971, p. 94. Dans ce même Testame'nt d'Abraham B 9.8 se trouve atussi l'une des toutes premières attestations d'un "lieu intermediaire" dans l'Au-delà (réservé à celui dont le's péchés étaiént a égalité ale'e se's bonne's actions), un lieu que l'eschatologie iranienne (qui le réserve aux âme's de ce's pe'r. somnes demt les actes meriteires e't le's péche's furent équivalents) appelle " hamiyastagan" (Arda Vira. Namag 6). un lieu qui recevara vers la fin du 12 siecle a Paris le nom de " Purgatorium " (ce que le (ioft. 1981. établit magistralement dans son étude dont la première centaine de pages appelle. cependant, de très séricuses réserves. un seul exemple: p. 9l est attribué à Paulin de Nole (mort en 431 ) une citation du Psaume 66 |Vulgate 6.5|, 12) : vu l'importance de l'interprétation des Psalumes dans la théologie des premiers siècles, les conclusions qu’on peut tirer d’un tel texte risque fort d’être faussées.

le destinataire de ces hommages a bien contribué a nourrir ce dossier, voir. par exemple Philonenho, 1986, 1992. 1994. Widengren. 1995. des siectes

(eci constitue une des caractéristiques du fonctionnement des textes apocalyptiques tout au long

Fntrant par Antioche, dont les relations avec la côte palestinienne jusqu en Egypte étatent permanentes. et de la se diffusant vers l'Est.

les nombreuses traditions apocalyptiques prêses à Ephrem pourraient constituer une confirmattion de cette vitalite continuelle

"La description de la porte du troisieme ciel, en IV. est intéressante à ee sujet. Ie voyageur rap-

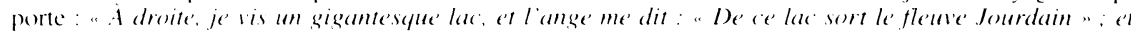
sur son rivage (il y al'ait) comme un prêtre érant, et l'ange' me dit : " "e'st Je'an le Baptiste! ". On pour rait se demander si l'idée d'une source céleste du Jourdain ne serait pas la marque laissée dans le texte par son passage dans une secte haptiste syrienne?). 
La même explication vaut pour d'autres textes qui pourraient trouver une solution à leurs difficultés dans ce même cheminement, des apocalypses", ou d'autres compositions qui souvent soulèvent des questions qui ne sont pas faciles à résoudre".

Une telle hypothèse, qui ouvre encore d'autres portes?, pourrait s’avérer extrêmement féconde et ne manquerait pas. jen suis persuadé, d'apporter des solutions nouvelles à de vieux problèmes.

\section{BIBLIOCRAPHIE}

Aerts - Kortekatas, 1998: W.J. Aerts- G.A.A. Kortekatas, Die Apokalypse des PséudoMethodius. Die älteste'n griechischen und lateinischen Überse'zungen IC.S.C.O. 569-570, Subsidia 97-981, Louvain. 1998.

Alexander, 1967 : Paul J. Alexander, The ()racle of Bablberk - The Tiburtine Sibyl in Greck Dress [Dumbarton Oaks Studies 10), Washington D.C.. 1967.

Alexander, 1985: Paul J. Alexander, The Byzantine Apocalyptic Tradition, Berkeley/Los Angeles/London, 1985.

Asin y Palacios, 1919: Miguel Asin y Palacios, La escatologia musulmana en la Divina Comedia, Madrid, 1919) (deuxièmé édition complétée 1943); l'ouvrage est redevenu d'actualité par une traduction anglaise condensée (de Harold Sutherland) Islam and the Divine' Comedy [Islam and the Muslim World 4], London, 1968.

Berger, 1976 : Klaus Berger, Die griechische Daniel-Diegese - Eine altkirchliche Apokalypse [Studia Post-Biblica 27], Leiden, 1976.

Böklen, 1902 : E. Böklen, Die Verwandtschaft der jüdisch-christlichen mit der parsischen Eschatologie, Göttingen, 1902.

Budge, 1915 : E.A.W. Budge, Miscellaneous Coptic Texs in the Dialect of Upper Egypt, London, 1915.

Carozzi, 1994 : Claude Carozzi, Le voyage de l'âme dans l'Au-delì d'après la littérature latine ( $V$-XIII siécles) |École Française de Rome 1891, Rome, 1994.

Charlesworth, 1983 : James H. Charlesworth, ed., The Old Testament Pse'udepigrapha, New York/London, 1983.

Dinzelbacher, 1973 : Peter Dinzelbacher, Die Jenseitshriicke im Mittelalter, Wien, 1973.

Dinzelbacher, 1989 : Peter Dinzelhacher, Mittelalterliche Visionsliteratur - eine Anthologie, Darmstadt, 1989.

Dupont-Sommer - Philonenko, 1987 : André Dupont-Sommer et Marc Philonenko, edd., Érits Intertestamentaires [Bibliothèque de la Pléiade 337|, Paris, 1987.

"Il me semblerait interessant dessayer de vérifier celle hypothese dans ke cas de l'Apocalypse" greceue d'Esdras (dont la langue est by/antine alors que le materiau semble bien plus ancien) voit l iexcellent état de la question dans Nuvolone. 1996: peut-etre aussi dans celui de la Premieré Apocalypse apocryphe de Jean.

Par exemple, les Paralipomenes de dereme dont certanes expressions fleuries trouvent leur explication dans une parenté de style avec les textes mandéens de Syrie, comme la fort bien montré Philonenko, 1996.

Ainsi. pour en revenir aux apocalypes by/antines " historiques", si l'on a pu vérifier que le Pséudo-Méthode grec a été traduit du syriaque. il n'en ést pas acquis pour atutant que lat version syriaque soit longinal : il pourrait dejat sagir diune traduction... 
Gidel, 1871: Charles Gidel. "litude sur une apocalypse de la Vierge ". Anmuctire de" l'Association pour l'encourageme'nt de's étude's greceque's en France 5, 1871, p. 92113.

Hage, 1974: W. Hage, Die griechische Baruch-Apokalypse |J.S.H.R.Z. 5.11, Giitersloh, 1974.

Homburg, 1903/1 : Rudolf Homburg. Apocalypsis Anastasiae' |Bibliotheca Teubneriana|. Lecipzig. 1903.

Homburg. 19()3/1 : Rudolf Homburg, "Apocalypsis Anastasiace ", Keitschrift fiur wissenschaftliche Theologie 46, 1903, p. 434-466.

Istrin, 1897: V. Istrin, L'apocalypse de Méthode de Patara et les visions apocryphes de Daniel dans le's littératures byzantine e't slano-russe |Recueils de la Société Impériale d'histoire et des antiquités russes, vol. 181 Études et vol. 183 Textesllen russel, Moscou, 1897.

James, 1897 : Montague Rhodes James, Apocrypha Anecdota // |Texts and Studies V,11, Cambridge, 1897.

Le Goff, 1981 : Jacques Le Goff, La naissance du Purgatoire, Paris, 1981.

Kmosko, 1931 : Michael Kmosko, "Das Rätgel des Pseudo-Methodius ", Bvzantion 6. 1931, p. 27.3-296.

Nau, 1914 : François Nau (1914), "Une deuxième apocalypse apocryphe de Jean ", Rev'ue Biblique 11, 1914, p. 209-221.

Nau, 1917 : François Nau 1917, «Révélations et légendes. Méthodius. - Clément. Andronicus. ", Journal Asiatique, 2" série, 9, 1917, p. 415-471.

Nuvolone, 1996 : Flavio Nuvolone, «Apocalypse d'Esdras grecque et latine, rapports et rhétorique ", Apocrypha 7, 1996, p. 81-108.

Onasch, 1957 : K. Onasch, «Paraskeva-Studien », Ostkirchliche Studien 6, 1957.

Pavlova-Sivanscaya, 1968 : M. Pavlova-Sivanscaya, "L’icône de Paraskeva-Piatnitsa entourée de 18 scènes de sa vie ". L'Art ancien russe. La culture artistique de Pskov len russel, Moscou, 1968, p. 127-138.

Philonenko, 1986 : Marc Philonenko, «Philon d'Alexandrie et l'“Instruction sur les deux Esprits », Hellenica et Judaica, Louvain-Paris, 1986, p. 61-68.

Philonenko, 1992: Marc Philonenko, «Un esprit ténébreux et puant (Alchimistes grecs 296,13)» (RAIBL, 1992, Paris, p. 651-662.

Philonenko, 1993 : Marc Philonenko, ed., Le trône de Dieu /Wissenschaftliche Untersuchungen zum Neuen Testament 69|, Tübingen, 1993.

Philonenko, 1994 : Marc Philonenko (1994), "Le Pont de l'Abîme ", in Le Signe, le Symbole et le Sacré [Cahiers internationaux de symbolisme, 77-79], 1994, p. $181-186$.

Philonenko, 1996 : Marc Philonenko, "Simples observations sur les Paralipomènes de Jérémie ", RHPR. 76, 1996, p. 157-177.

Reinink, 1993 : G.J. Reinink, Die strische Apokalypse des Pseudo-Methodius [C.S.C.O. 54()-541/Scriptores Syri 22()-2211, Louvain, 1993.

Radermacher, 1898 : L. Radermacher, "Anonymi Byzantini de caelo et infernis epistula", Studie'n zur (ieschichte der Theologie und der Kirche 3, 1898, p. 1-30.

Ryden, 1994 : Lennart Ryden. "The Andreas Salos Apocalypse", Dumbarton Oaks Papers 28, 1994. p. 197-261.

Sackur, 1898 : E. Sackur. Sibyllinische Texte und Forschungen, Halle, 1898.

Schmidt, 1971 : Francis Schmidt, Le' Téstament d'Abraham (Thèse dactylographiée Université des Sciences Humaines de Strasbourg, Sciences religieuses), Strasbourg. 1971. 
Silverstein. 1935: Theodore Silverstein. Visio sancti Pauli Studies and Documents IVI. London. 1935.

Suermann. 1985: Harald Suermann. Die (ie'schichtsthesolegische Reaktion auf die cinfallende'n Mussime in de'r edessenischen Apokalyptik des 7. Jahrhunderts |turopäische Hochschulschriften Reihe 23. Band 2561. Franfurt/Berlin/New York. 1985.

Vaillant. 1952: André Vaillant, Le Livere des secrets d'Hénoch lTextes publiés par I'Institut d'Études slaves IV|. Paris, 1952.

Vassiliev, 1893: A. Vassiliev, Anecdota (iraeco-byamtina I, Moscou, 1893.

Verhelst, 198.3: D. Verhelst, "Scarpsum de dictis sancti Efrem prope fine mundi ", Pascua Mediaevalia Studies roer Prof. Dr. J.M. de Simet IMediatevalia Lovaniensia I,10). Leuven, 1983. p. 518-528.

Widengren, 1957 : (ieo Widengren, "Quelques rapports entre Juifs et Iraniens a l'époque des Parthes ". Vetus Téstumentum. Suppleme'nt IV, 1957, p. 197-241.

Widengren, 1995: (ieo Widengren, Anders Hultgard, Marc Philonenko, edd.. Apocalyptique iranienne et dualisme qoumrânie'n | Recherches intertestamentaires 21, Paris, 1995. 